\title{
High-energy dose of therapeutic ultrasound in the treatment of patellar tendinopathy: protocol of a randomized placebo- controlled clinical trial
}

Julio Fernandes de Jesus ${ }^{1,2,3^{*}}$, Tadeu Aldrovando Brihy de Albuquerque ${ }^{3,4}$, Leandro Girardi Shimba ${ }^{5}$, Flavio Fernandes Bryk ${ }^{3}$, Jill Cook ${ }^{6}$ and Carlos Eduardo Pinfildi ${ }^{2,7}$

\begin{abstract}
Background: Patellar tendinopathy is an extremely debilitating condition and its treatment usually requires a combination of clinical approaches. Therapeutic ultrasound (TUS) is one of the most available electrophysical agent in rehabilitation settings; however, there is also a lack of high-quality studies that test different dosimetric aspects of TUS. Thus, the purpose of this study is to evaluate the short-, medium-, and long-term effects of the combination of high-energy TUS with a rehabilitation program for patellar tendinopathy.

Methods: This will be a randomized, placebo-controlled trial with blinding of patients, assessors, and therapist. The setting is an outpatient physical therapy clinic. We will recruit 66 participants (male and female) aged between 18 and 40 years and presenting with patellar tendinopathy. A treatment combining high-energy dose TUS and a rehabilitation program for patellar tendinopathy will be delivered twice a week for 8 weeks. The control group will receive the same treatment, but with a placebo TUS. The effectiveness of the intervention will be measured at the beginning (baseline), midpoint (4 weeks), and end of treatment (8 weeks), as well as at 3- and 6-months posttreatment. Primary outcomes will be pain intensity (visual analogue scale, VAS), and VISA-P questionnaire and primary time points will be baseline (T0) and the end of the program (T2). Also, IPAQ-short form questionnaire, muscle strength (manual dynamometry), 2D kinematics, pain pressure threshold (PPT) algometry, thermography, and magnetic resonance imaging (MRI) will be collected.
\end{abstract}

Discussion: TUS will be applied in an attempt to enhance the results obtained with the rehabilitation program proposed in this study, as well as stimulate some repair responses in individuals undergoing treatment for patellar tendinopathy, which in turn may optimize and improve treatment programs for patellar tendinopathy as well as to establish new guidelines for the application of TUS.

Trial registration: This study was prospectively registered at April-3rd-2018 and updated at September-1st-2019 in the Brazilian Registry of Clinical Trials (REBEC) under the registration number: RBR-658n6w.

Keywords: Knee, Patellar Tendinopathy, Therapeutic ultrasound, Rehabilitation

\footnotetext{
*Correspondence: juliofernandes85@gmail.com

'Human Movement Science and Rehabilitation Postgraduate Program, Universidade Federal de São Paulo - UNIFESP, campus Baixada Santista, Santos, SP 11015-029, Brazil

2Physical Agents and Rehabilitation Research Group GPRAE, Universidade

Federal de São Paulo - UNIFESP, campus Baixada Santista, Santos, SP

11015-029, Brazil

Full list of author information is available at the end of the article
}

(c) The Author(s). 2019 Open Access This article is distributed under the terms of the Creative Commons Attribution 4.0 International License (http://creativecommons.org/licenses/by/4.0/), which permits unrestricted use, distribution, and reproduction in any medium, provided you give appropriate credit to the original author(s) and the source, provide a link to the Creative Commons license, and indicate if changes were made. The Creative Commons Public Domain Dedication waiver (http://creativecommons.org/publicdomain/zero/1.0/) applies to the data made available in this article, unless otherwise stated. 


\section{Background}

The patellar tendon is part of the knee extensor mechanism and tolerates heavy loads during lower limb movements [1]. Tendon consists of tenocytes, parallel collagen fibers, and extracellular matrix, which enable it to transfer, store, and dissipate energy [2]. Despite its structure, excessive cyclical overload can cause pathology and consequently pain and functional limitations, triggering patellar tendinopathy $[3,4]$.

Patellar tendinopathy is a debilitating condition that affects professional [5] and recreational [6] athletes. It is characterized by pain at the patella's apex $[7,8]$ and presents with tendon pathology [9-11], reduced tendon stiffness [12], muscle recruitment inhibition, biomechanical impairments [13-15], and proprioceptive deficits $[16,17]$.

Generally, the rehabilitation of this condition is focused on exercise based-programs [18], especially multiphase and individualized-progressive therapeutic exercises [4]. However the management of patellar tendinopathy remains complex and difficult since a combined clinical approach that takes into account tendon pathology [19] and functional impairments [4, 20] has not yet been investigated.

Therapeutic ultrasound (TUS) is one of the most available electrophysical agent in physical therapy settings $[21,22]$. Furthermore, presents some positive results about tendon pathology modulation and repair process in some experimental animal trials [23, 24]. However, the pathophysiological process of patellar tendinopathy in humans differs substantially from the experimental models [25] and making difficult translate the dosimetry from experimental animal trials to humans.

Besides that, it is still unclear which ultrasound dose is beneficial for the treatment of tendinopathy. Alexander et al. [26] showed that higher doses of ultrasound energy improved pain in individuals with shoulder calcific tendinitis. In contrast, Warden et al. [27] found that lower doses of ultrasound was no better than placebo for the treatment of individuals with patellar tendinopathy. In this context, higher doses of ultrasound might be necessary in order to produce significant improvements in patients with tendinopathy.

The etiology of patellar tendinopathy involves tendon overload [28], thus clinicians should consider general exercises to enhance kinetic chain energy dissipation and prioritizing local interventions to modify mechanical properties of the tendon [29]. Because of that, TUS dosimetry features could optimize the current clinical approach, once patelar tendinopathy has a potential to become recalcitrant [30]. However, there are no studies that have tested high-energy doses of TUS combined with exercise-based rehabilitation programs for patellar tendinopathy.
Thus, this placebo-controlled clinical trial aims to evaluate pain, motor function, and muscle strength of the lower limbs of individuals with patellar tendinopathy who complete a rehabilitation program combined with high-energy TUS.

\section{Methods \\ Aim}

The aim is to compare the effects in short-, medium-, and long-term of high-energy TUS or its placebo with a rehabilitation program for patellar tendinopathy.

\section{Study design}

This is a protocol of an ongoing randomized, placebocontrolled trial with blinding of patients, assessors, and therapist.

\section{Study settings}

The participants will be treated in an outpatient physical therapy clinic from São Paulo, Brazil. This project was approved by the Research Ethics Committee of the Federal University of São Paulo (UNIFESP) under registration number 2.351.182. To participate in this study all participants will give consent and sign the informed Consent Form (Additional file 2), before baseline assessment. It was also prospectively registered at April-3rd-2018 and updated at September-1st-2019 in the Brazilian Registry of Clinical Trials (REBEC) under the registration number: RBR-658n6w. If there is a need to change the protocol, the change will be immediately reported to the Research Ethics Committee and carried out at the registration site.

There aren't any publications or submissions containing any of the results of this study.

\section{Participants}

We will recruit individuals of both sexes who are seeking treatment for patellar tendinopathy in primary and secondary health services (new presentations), aged between 18 and 40 years-old, with localized pain (pain map will be marked by the participants) at the inferior patellar pole/superior aspect of patellar tendon with load (single leg decline squat) [31], pain when jumping/landing, running or abruptly changing direction, and with a score of less than 80 on the Victorian Institute of Sports Assessment - Patella (VISA-P) questionnaire [32-34].

Participants will be excluded if they present a history of nervous and/or musculoskeletal injuries in the low back spine and/or lower limbs in the last 6 months [35], signs and/or symptoms of other diseases and/or another disorders affecting the knees [27], including systemic and rheumatic diseases and/or pre-existing dysfunctions (clinically evaluated and detected) [36], previous history of knee or patellar tendon surgery [27, 37], use of intraarticular medications in the knee in the last 6 months 
[27], or pregnancy. A single physician - who consults at primary and secondary health services will standardize recruitment and determine eligibility, based on clinical history testing, along with imaging examination of potential participants.

\section{Randomization and masking}

All participants will be informed about the study procedures and then they will be randomly allocated to one of the intervention groups (active TUS or placebo TUS). The researcher not involved with the data collection will allocate the participant using numbers ( 1 or 2 ) placed according to the sequence presented in the plan and sealed in opaque envelopes without stratifications or covariates consideration. The randomization sequence was created at http://www.randomization.com, in a single block with two distinct labels: 1 and 2 (referring to the two groups of this study).

Participants and the therapist will be blinded throughout the treatment. To ensure blinding, two identical TUS machines will be used, however one will have its software modified by the manufacturer to simulate sound wave emission ( $30 \mathrm{~s}$ of stimulation followed by an undetectable pause in the sound wave emission) and only a researcher who is not involved in collecting data knows if the equipment is active or placebo. Furthermore, and to keep the therapist blinded, the sessions outcomes: visual analogue scale (VAS) - VAS-usual and -irritative, pain pressure threshold (PPT)-algometry, and thermography will be collected by an assessor (not the therapist) who will be unaware of each participant's group allocation; and the treatment room will be set up by a research assistant with only one TUS equipment (in agreement with participant group allocation: active or placebo). The participants will be informed before the treatment about the characteristics of the rehabilitation program and asked to not discuss the TUS application with the therapist and assessor researchers.

\section{Intervention}

Participants will take part in the exercise program (Tables 1 and 2) and TUS application (active or placebo). If there are bilateral complaints, the most symptomatic knee will receive TUS but both lower limbs will receive exercises.

The TUS dosimetric settings $[26,38,39]$ are described in Table 3 and the applications will be carried out twice a week during the treatment period ( 8 weeks - 16 sessions) and always at the beginning of the session (before exercise). Individuals will be placed in supine during the application with the knee flexed at approximately $20^{\circ}$ and stabilized by a positioning cushion.

TUS will be applied in the patellar tendon region (Fig. 1), with full contact between the TUS transducer and the treatment area. The gel will be used as the conduction agent and circular movements of the head device will be performed at a slow speed, approximately 4 $\mathrm{cm} / \mathrm{s}$.

A program of therapeutic exercises was developed based on clinical features [40-42] and specific strategies for patellar tendinopathy management $[4,43]$ and also in similar exercises $[44,45]$ and protocols applied in other overload conditions [46]. This program will be delivered in four phases, each one containing 2 weeks duration [20, 35, 47] (Tables 1 and 2) and the standardized sessions will be performed in individually and will be supervised by the same physical therapist that is also responsible for all TUS applications. The participants will be instructed not to use other interventions, but will be encouraged to stay active in their physical activities or sports during this program, however respecting their level of function and pain. This information will be given to the participants at the initial examination.

\section{Outcome measure}

Pain intensity will be measured using the Visual Analogue Scale (VAS) [48, 49]. This outcome will be always based on routinely presented pain (VAS-usual) and pain during the eccentric decline squat (VAS-irritative at pre-TUS and post-TUS) [27, 50]. Pain, dysfunction and the level of physical activity will be measured with the VISA-P questionnaire [34] and the International Physical Activity Questionnaire-Short Form (IPAQ-short form) [51], respectively.

The trunk and lower limbs kinematics will be assessed with two-dimensional (2D) kinematic analysis, once patellar tendinopathy may cause kinematic changes at hip [33, 52], knee [53, 54] and foot [53, 54] levels during landings the 2D-kinematics analysis will be applied in attempt to detect lower limb and trunk kinematic patterns on different types and intensities of landing tasks in both groups.

The acquisition of these images will be carried out with two cameras Optitrack (NaturalPoint Inc., Corvallis, OR, USA) with 0.3 MP resolution and capture rate of 100 FPS, two cameras (color video capture) Logitech Model: C920 (Logitech, Newark, CA, USA) with 3 MP resolution and capture rate of $30 \mathrm{FPS}$ and with the software myoVIDEO - MR3 3.6.43 (Noraxon USA Inc., Scottsdale, AZ, USA). Reflective markers will be applied on the acromion bilaterally, manubrium, xiphoid process, antero-superior iliac spines, patellar bases, ankle joint lines, major trochanters, lateral femoral condyles of the knees, lateral malleoli, intermediate third of the fibula diaphysis, and on the tuberosity of the fifth metatarsals $[55,56]$.

The following movements will be verified and quantified in the frontal plane: trunk inclination, pelvic tilt, hip 


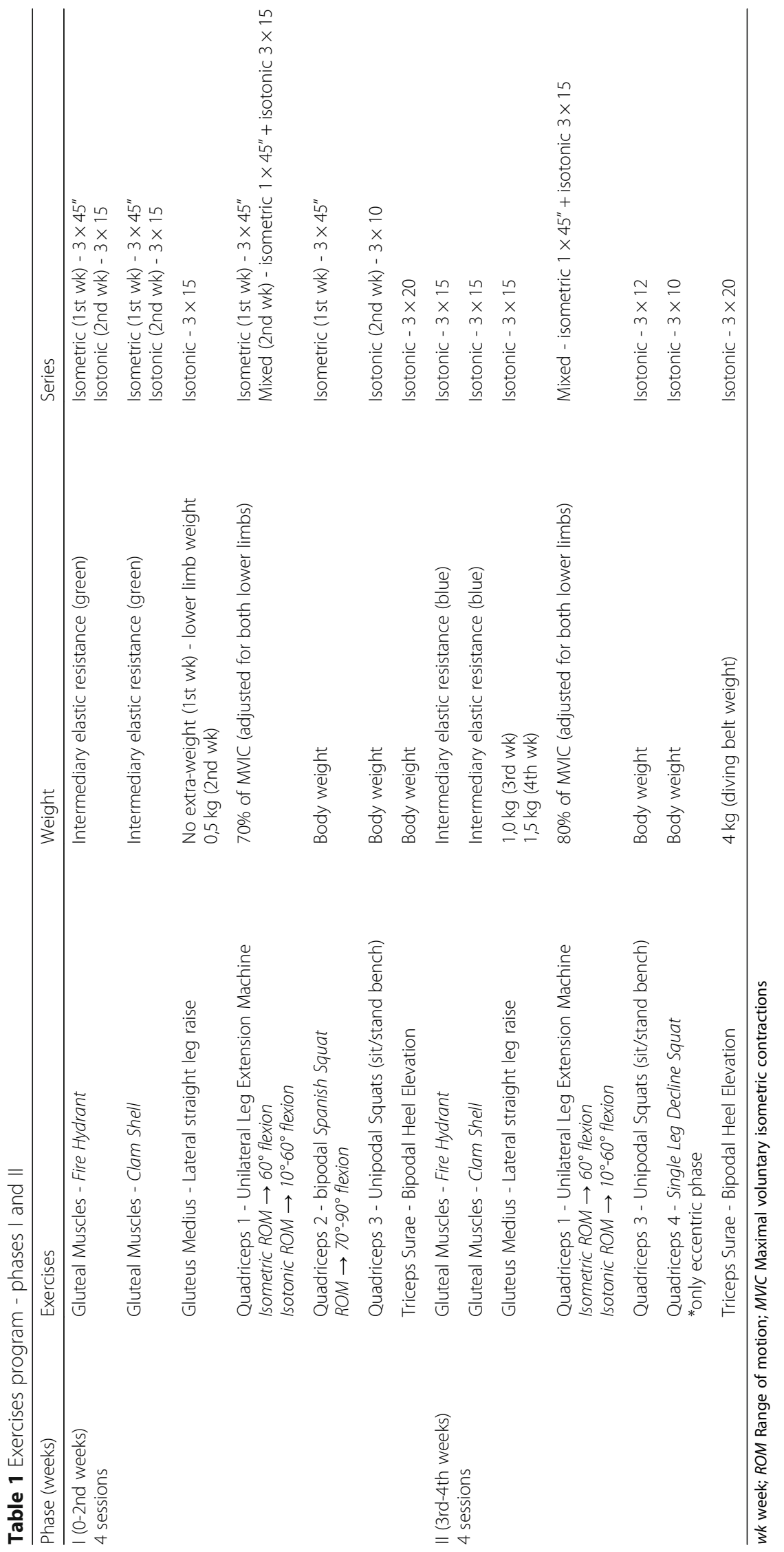




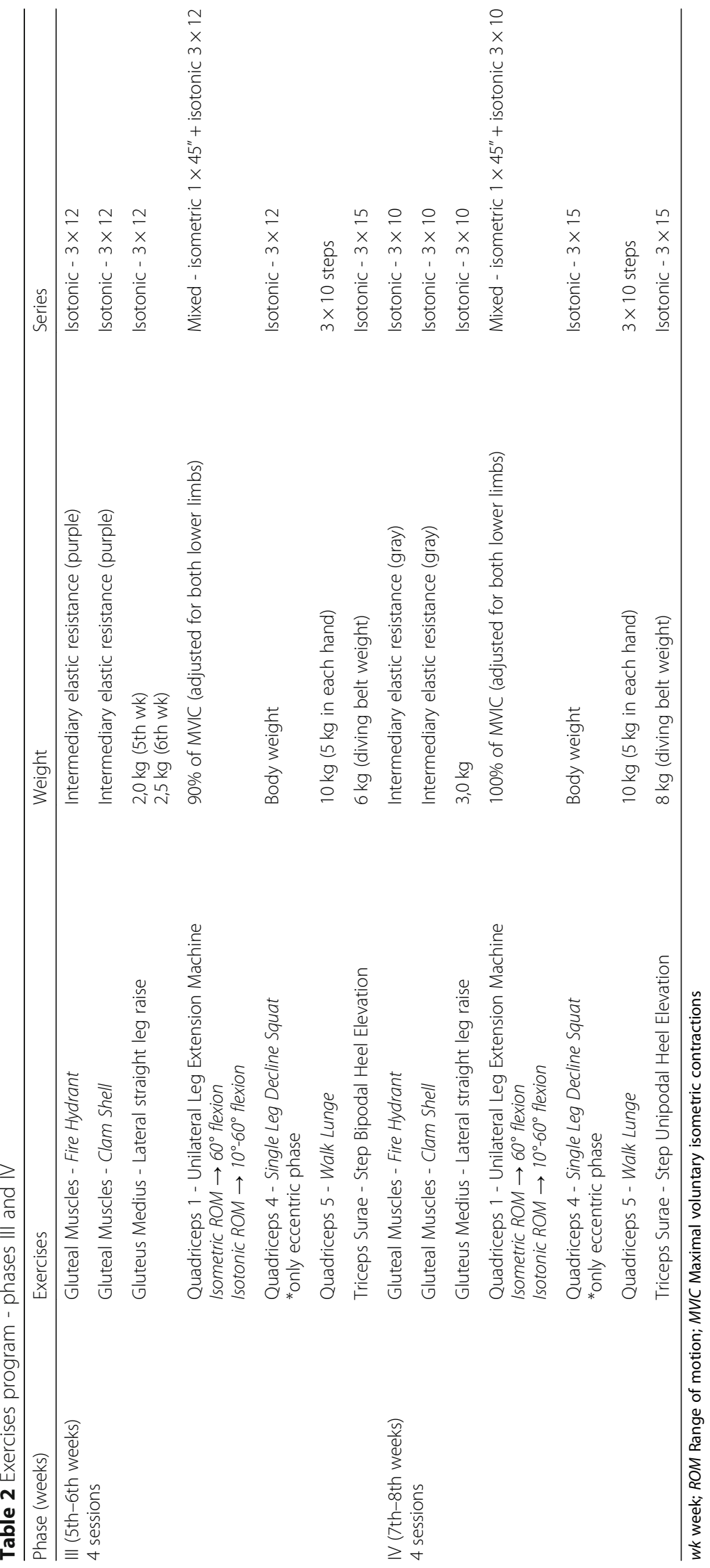


Table 3 Therapeutic ultrasound (TUS) dosimetric parameters

\begin{tabular}{ll}
\hline Parameters & Adjusts \\
\hline Frequency & $1 \mathrm{MHz}$ \\
Mode & Continuous \\
Effective radiation area (ERA) & $7 \mathrm{~cm}^{2}$ \\
Spatial average - Temporal peak (SATP) & $1,2 \mathrm{~W} / \mathrm{cm}^{2}$ \\
Time (t) & $8 \mathrm{~min}$ \\
Energy (E) & $4032 \mathrm{~J} /$ per application \\
Beam non-uniformity ratio (BNR) & $3: 1$ \\
Application speed & Slow $( \pm 4 \mathrm{~cm} / \mathrm{s})$ \\
\hline
\end{tabular}

adduction, and knee valgus; and in the sagittal plane: trunk flexion, hip flexion, knee flexion, and ankle dorsiflexion. The time to acquire the peak knee flexion will also be quantified. The motion cameras for the frontal and sagittal planes will be placed $3.5 \mathrm{~m}$ and $3.0 \mathrm{~m}$ from the participant, respectively, for data collection on Single Leg Squat (SLS) $[55,57]$ and Drop Vertical Jump Test Double and Single Leg (DVJ-DL and DVJ-SL) [55, 58]. For the Single Hop Test for Distance (SHTD) [59], the cameras will be placed $3.7 \mathrm{~m}$ and $3.0 \mathrm{~m}$ from the participant for frontal and sagittal plane data collection, respectively. The motion analyses will be performed at peak knee flexion, as this moment will be the reference for all measurements.

The strength (maximum voluntary isometric contraction, MVIC) of the gluteus maximus [42, 60] (hip extension), gluteus medius [61] (hip abduction), quadriceps femoris

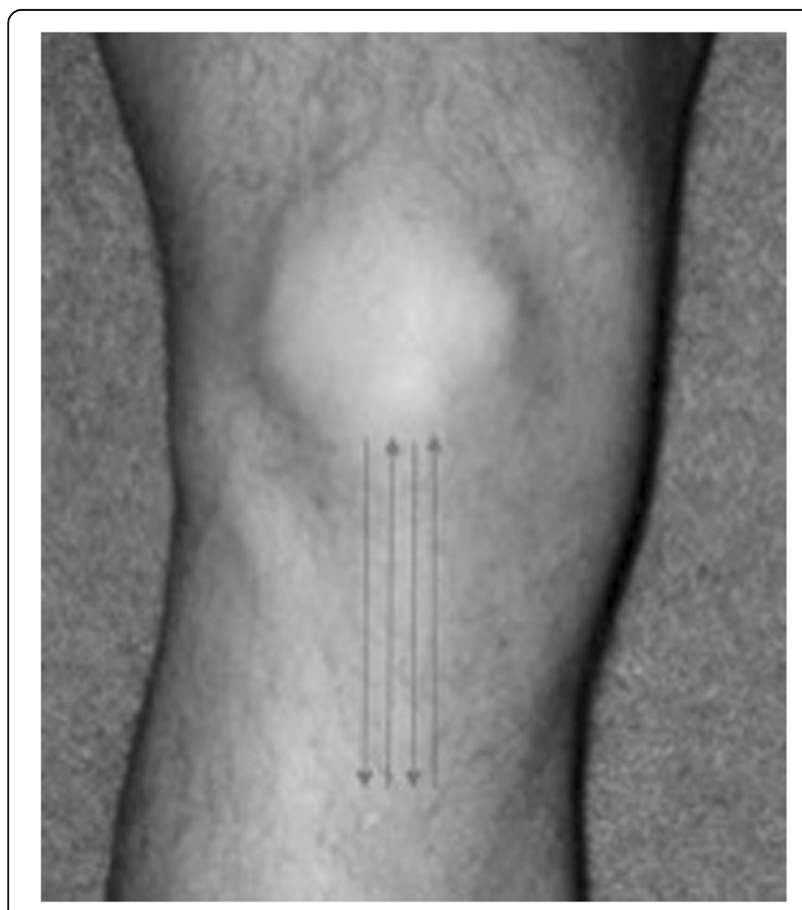

Fig. 1 Local of TUS application (patellar tendon)
[59] (knee extension), and triceps surae [42] (ankle plantar flexion) will be assessed with a handheld dynamometer (Manual Muscle Tester, model 01163, Lafayette Instrument Company, Lafayette, IN, USA) [62]. A nylon belt will be positioned perpendicular to the dynamometer during the tests in order to stabilize the dynamometer and to resist the force generated by the participant. Two 5-s MVIC will be performed with a 30-s interval between contractions [59]. The average strength of each muscle - obtained in kilogram/force $(\mathrm{KgF})$ - will be normalized by the body weight of each participant, using the following formula: (muscle strength kg/ body weight kg) X 100 [59].

Pain pressure threshold (PPT)-algometry (PPT - algometer model: DD-2000, Instrutherm, São Paulo, SP, Brazil) of the patellar tendons will be performed at T0, $\mathrm{T} 1$ and $\mathrm{T} 2$ evaluations time-points and at every session - before and after the TUS application $\left( \pm 8^{\prime}\right.$ interval between PPT evaluation). For this measurement, the participant will be positioned in supine, with the knee flexed $\left( \pm 20^{\circ}\right)$ and stabilized with a positioning cushion and as soon as the individual experienced a painful/discomfort sensation he/she will say "stop"; the PPT algometer will be immediately released and the force (in Newtons) will be read from the display. The data will be collected twice (the average will be used) directly on the patellar tendon (just below the apex of the patella) [63].

There is more specific information about 2D kinematic analysis, muscle strength dynamometer and PPT algometry at Additional file 1.

Thermography of the patellar tendons will be assessed with the thermal camera FLIR C2 (FLIR Systems Inc., Danderyd, Sweden), which has a resolution of $320 \times 240$ pixels and in accordance with international recommendations [64]. Two images will be captured, and the average of the two images will be used. Before collecting data on thermography, participants will remain for $10 \mathrm{~min}$ in a room at ambient temperature. The camera will be positioned perpendicular to the patellar tendon at a distance of approximately $30 \mathrm{~cm}$ [65-68].

Possible tissue changes of the patellar tendons or adverse events will be assessed by magnetic resonance imaging (MRI) at two separate time points: before (T0; initial MRI) and after the treatment program (T2-final MRI). The images will be acquired in a magnetic field of $1.5 \mathrm{~T}$ (high field). The MRI protocol will consist of proton-density (PD) T2-weighted fast spin-echo (FSE) sequences acquired in the coronal, axial, and sagittal planes, with pulse sequence and cuts of $3 \mathrm{~mm}$ thickness, $0.3 \mathrm{~mm}$ inter-cut space, repetition time (TR) of $3760 \mathrm{~ms}$, echo time (TE) of 16.25 $\mathrm{ms}$ and $97.54 \mathrm{~ms}$, respectively, without averages, field of view (FOV) of 150 X 150 X $106 \mathrm{~mm}, 32$ cuts with acquisition matrix of $256 \mathrm{X} 256$, resulting in 0.586 
$\mathrm{mm}$ of plane resolution [69, 70]. The following characteristics will be assessed: intra-tendinous signal, involvement to the retropatellar region (Hoffa's fatpad), tendon volume, the area of the maximum transverse section of the tendon, maximum antero-posterior tendon diameter, and signal intensity [70, 71]. A physician will perform the MRI assessments with clinical experience in imaging analysis, with no knowledge of group allocation or MRI order (initial or final).

\section{Data collection}

After recruitment and before the beginning of treatments, demographic/personal data, clinical history (duration of symptom), and patellar tendon morphological characteristics (initial magnetic resonance imaging MRI) will be collected.

The outcomes of this study (Table 4) will be collected in five time-points: before starting the program (baseline - T0), in the middle (after 4 weeks/8 sessions - T1), in the end (after 8 weeks $/ 16$ sessions - T2), three and 6 months after the end of the program (follow-ups T3 and $\mathrm{T} 4$, respectively). The T3 and T4 data collection will be performed by mobile text messages, telephone contact or mail (depending on participant preference). However, the primary time points will be baseline (T0) and the end of the program (T2).

Beyond that, in all sessions will be collected VAS[48, 49]-usual, VAS-irritative: pre- and post-TUS [4]; and thermography: pre-TUS, post-TUS, pre-quadriceps exercises, and at the end of the session. The MRI will only be acquired at the beginning and at the end of treatment program.

An assessor who will be unaware of each participant's group allocation will collect all these outcomes. Figure 2

Table 4 Outcome measured and time-points

\begin{tabular}{|c|c|c|c|c|c|c|}
\hline \multirow[t]{2}{*}{ Outcomes } & \multicolumn{6}{|c|}{ Data collection time-points } \\
\hline & $\overline{\mathrm{T} 0^{\mathrm{a}}}$ & All sessions & $\mathrm{T} 1$ & $\mathrm{~T} 2^{\mathrm{a}}$ & T3 & $\overline{\mathrm{T} 4}$ \\
\hline \multicolumn{7}{|l|}{ Primary } \\
\hline 1. VAS & $x$ & $x$ & $x$ & $x$ & & \\
\hline 2. VISA-P & $x$ & & $x$ & $x$ & $x$ & $x$ \\
\hline \multicolumn{7}{|l|}{ Secondary } \\
\hline 3. IPAQ-short form & $x$ & & $x$ & $x$ & $x$ & $x$ \\
\hline 4. PPT algometry & $x$ & $x$ & $x$ & $x$ & & \\
\hline 5. Thermography & $x$ & $x$ & $x$ & $x$ & & \\
\hline 6. Muscle strength & $x$ & & $x$ & $x$ & & \\
\hline 7. $2 \mathrm{D}$ kinematics & $x$ & & $x$ & $x$ & & \\
\hline 8. MRI & $x$ & & & $x$ & & \\
\hline
\end{tabular}

a Primary time-points

VAS Visual analogue scale; VISA-P Victorian Institute of Sports AssessmentPatella; IPAQ-short form International Physical Activity Questionnaire-Short Form; PPT Pain Pressure Threshold; 2D kinematics two-dimensional kinematic analysis; MRI Magnetic resonance images shows the flowchart with a summary of experimental procedures and the participation of individuals, according to the Consolidated Standards of Reporting Trials (CONSORT) [72].

\section{Data analysis, monitoring, and auditing}

The sample size was calculated using the primary outcome variables: VAS and VISA-P questionnaire. A higher number of participants per group was required when the calculation was performed using data from the VISA-P questionnaire. For the calculation, the minimum detectable difference (MDD) considered was 12.2 points [34], with an average of the standard deviations of 16.2 points [27], resulting in 28 participants per group for an $\alpha$-error of 0.05 and a $\beta$-error of $80 \%$. Thus, 33 participants per group (assuming 15\% of possible sample losses) will be selected.

The head-coordinator (CEP) of the present study will be the responsible for the monitoring and auditing process of all stages related to this clinical trial, as well as be responsible for terminate it. These processes will be performed at regular times by personal visiting of data setting collection and checking the data bank archive, and if some irregularity or adverse event is detected; the trial will be interrupted.

All assessment forms will be scanned and stored in a safe place, and the data tabulation will be stored on the study's computer and on pen drives to avoid losses. The participants will be coded by numbers to maintain the confidentiality of their personal data, which will not be disclosed. One of the researchers (CEP) will certify the data collection stage. The final results of the study will only be revealed upon completion of all follow-ups, and only the authors of the research will have access to the data prior to publication in a peer-review journal. The data will be entered twice to avoid tabulation errors, and the statistician will receive the coded data and will be blind to group allocation.

The statistical analysis will be conducted following the principles of intention-to-treat analysis [73]. The normality of the data will be tested with visual inspection of histograms and the characterization of participants will be calculated using descriptive statistics. Improvements from baseline (T0) until the rehabilitation program final time-point (T2) will be assessed with a mixed model ANOVA (split-plot ANOVA). The differences between groups (treatment effects) and their respective confidence intervals $(95 \% \mathrm{CI})$ will be calculated for all outcomes, using mixed linear models for the interaction of groups by time. These analyses will be carried out using statistical software (Statistical Package for Social Sciences (SPSS) version 15.0). 


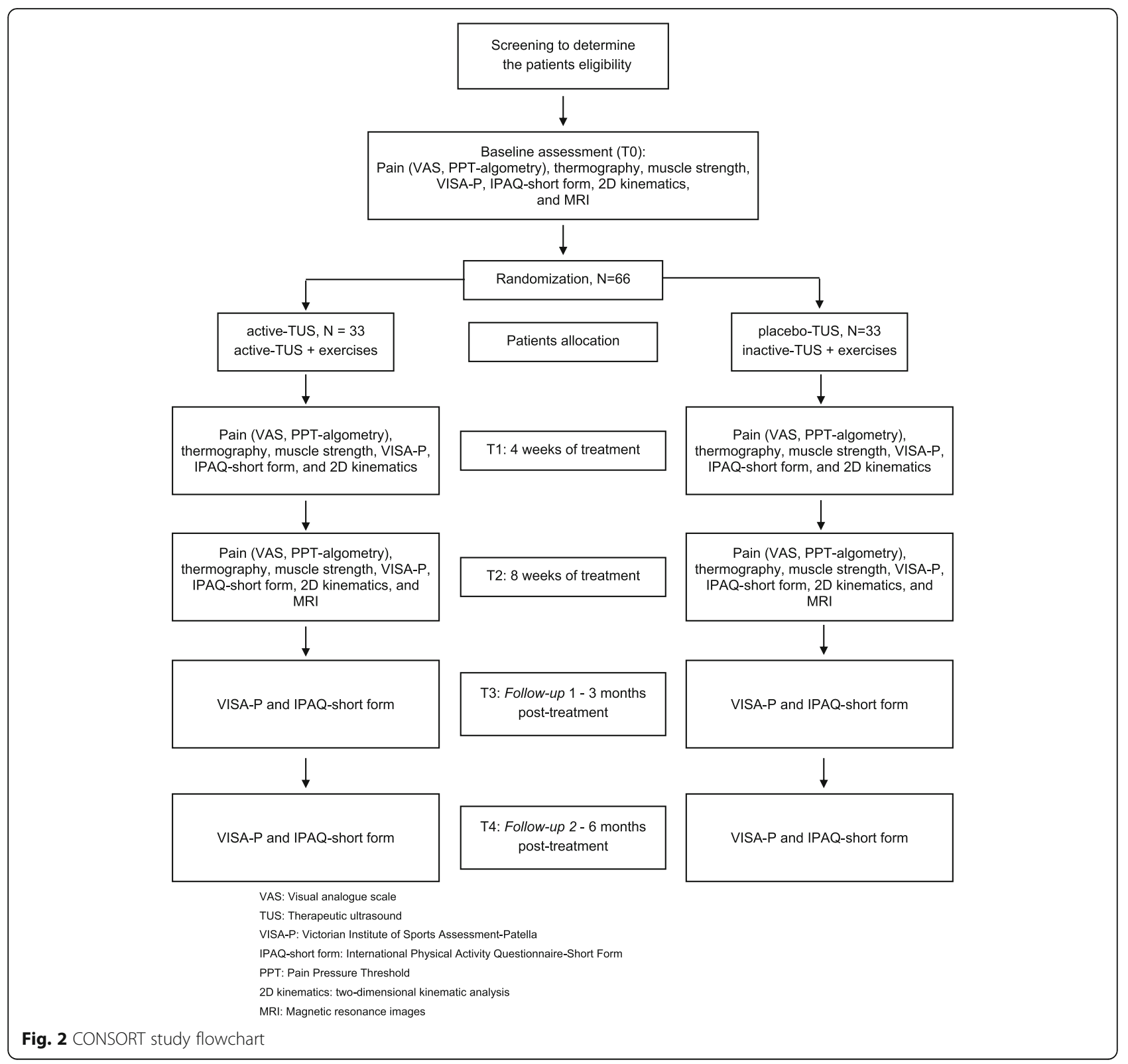

\section{Discussion}

This randomized, placebo-controlled, blinded clinical trial aims to examine the effectiveness of the combination of high-energy dose of TUS and a multiphase exercise rehabilitation program for patellar tendinopathy and determine whether TUS influences pain modulation and other outcomes such as muscle strength, lower limb motor function and morphological tendon characteristics.

Patellar tendinopathy is a condition with difficult clinical management due to its multifactorial etiology [74, 75]. Individuals suffering from patellar tendinopathy present with structural, biochemical and consequently functional changes that lead to load absorption and propagation deficits, and pain [76]. In addition, these changes hamper tissue repair and tend to cause perpetuation of the condition [28]. The outcomes chosen for this study were based on these changes with the aim of understanding the possible pathways of TUS in the modulations of the signs and symptoms in individuals with patellar tendinopathy.

Therapeutic exercises are the most researched interventions for the treatment of patellar tendinopathy, especially the eccentric decline squat, as it loads the injured patellar tendon [29]. It is possible that pain reduction improves muscle recruitment and consequently modulates lower limb biomechanics [77], promoting functional optimizations. However, therapeutic exercises in isolation do not always solve all of the complex 
symptoms of these patients, especially very painful symptoms and those of high-performance athletes during sports seasons [31].

Thus, TUS may enhance the results obtained with the rehabilitation program proposed in this study, as well as stimulate some repair responses [23, 24] in individuals undergoing treatment for patellar tendinopathy. The current literature shows increases in patellar tendon temperature after the application of TUS [78], an effect that could raise the pain threshold [79] and improve collagen extensibility [80]. These effects could favor the viability of the proposed exercises, optimizing the results. The add-on (chronic) effect promoted by high-energy TUS [81] could also influence the tissue structure of the tendons, making them more resistant and better able to absorb and dissipate energy, thus improving function in individuals with patellar tendinopathy.

Although there is evidence in the literature showing a lack of clinical benefits from TUS in the treatment of patellar tendinopathy [27], there is a lack of research with good methodological design testing the use of highenergy TUS combined with rehabilitation programs for this clinical condition. In addition, the lack of positive TUS results for the treatment of the most diverse conditions has been related to dosimetric errors [82].

In this context, Alexander et al. [26] demonstrated that doses of $2250 \mathrm{~J}$ (J) of energy (per treatment session) promoted improvement in pain in individuals with painful shoulders, while doses of $750 \mathrm{~J}$ or less rarely generated positive results. The treatment of elbow tendinopathy and calcaneal tendinopathy with a TUS dose of $18,250 \mathrm{~J}$ promoted significant improvement in pain and function [83]. Similarly, the application of doses of $1290 \mathrm{~J}, 2580 \mathrm{~J}$, and $11,250 \mathrm{~J}$ was effective for the treatment of calcific tendonitis of the shoulder, resulting in lower pain rates, improved motor function, and regression of pathological calcification [38, 39].

Due to these characteristics, the TUS dose will be $4032 \mathrm{~J}$ per application combined with a rehabilitation program based on current and scientifically developed clinical guidelines $[4,40-42]$. The dosage for the application of TUS is one of the most difficult factors to translate from animal studies to the clinical setting. The results from the animal studies open the way for the investigation of the effects of TUS in humans; however, the dosimetric factor is complex and there is no direct correlation between animals and humans. Therefore, the energy parameter $(\mathrm{J})$ is being used as a facilitator to the replication of these parameters.

The current study has a high methodological quality, as it is a prospective, randomized, and controlled clinical trial. The blinding of assessors, therapists, and patients, who will not have access to the characteristics of the interventions during the study, yields greater methodological relevance.
Finally, the sample size was calculated with the purpose of providing adequate statistical power and sensitivity to detect differences in the primary and secondary outcomes. Therefore, the design features of the current study allow a relevant contribution to evidence-based practice, particularly as concerns the clinical management of patellar tendinopathy with the use of TUS.

\section{Supplementary information}

Supplementary information accompanies this paper at https://doi.org/10. 1186/s12891-019-2993-2.

Additional file 1. Additional informations of 2D kinematic analysis, muscle strength dynamometer, and PPT-algometry.

Additional file 2. Consent statement.

\section{Abbreviations}

2D: Two-dimensional; DVJ-DL: Drop Vertical Jump Test - Double Leg; DVJSL: Drop Vertical Jump Test - Single Leg; FOV: Field of view; FSE: Fast spinecho; ICC: Intraclass correlation coefficient; IPAQ-short form: International Physical Activity Questionnaire-Short Form; J: Joules; KgF: Kilogram/force; MDD: Minimum detectable difference; MRI: Magnetic resonance images; MVIC: Maximal voluntary isometric contractions; PD: Proton-density; PPT: Pain pressure threshold; REBEC: Brazilian Registry of Clinical Trials; SHTD: Single Hop Test for Distance; SLS: Single Leg Squat; SPSS: Statistical Package for Social Sciences; TE: Echo time; TR: Repetition time; TUS: Therapeutic ultrasound; VAS: Visual analogue scale; VISA-P: Victorian Institute of Sports Assessment Questionnaire, Patellar

\section{Acknowledgments}

Not applicable.

\author{
Sponsor contact information \\ Sponsor: Universidade Federal de São Paulo - UNIFESP; campus Baixada \\ Santista. \\ Contact name: Carlos Eduardo Pinfildi. \\ Adress: Rua Silva Jardim, 136 - Vila Matias, Santos - SP, 11015-020. \\ Telephone: + 55 133,229-0100. \\ Mailing adress: cepinfildi@hotmail.com
}

\begin{abstract}
Author's contributions
JFJ - Conception, design of work, acquisition and analysis of data; TABA Acquisition and analysis of data; LGS - Design of work and acquisition of data; FFB - Conception and analysis of data; JC - Design of work and analysis of data; CEP - Conception, design of work, and analysis of data. All authors read and approved the final version of the above-referenced manuscript.
\end{abstract}

\section{Funding}

Not applicable.

\section{Availability of data and materials}

The datasets that will be used and/or analyzed during the current study will be available from the corresponding author on reasonable request.

\section{Ethics approval and consent to participate}

The present study was approved by the Research Ethics Committee of the Federal University of São Paulo (UNIFESP) under registration number 2.351.182. To participate in this study all participants will give consent and sign the informed Consent Form, before baseline assessment.

Consent for publication

Not applicable.

Competing interests

The authors declare that they have no competing interests. 


\section{Author details}

'Human Movement Science and Rehabilitation Postgraduate Program, Universidade Federal de São Paulo - UNIFESP, campus Baixada Santista, Santos, SP 11015-029, Brazil. 'Physical Agents and Rehabilitation Research Group GPRAE, Universidade Federal de São Paulo - UNIFESP, campus Baixada Santista, Santos, SP 11015-029, Brazil. ${ }^{3}$ Functional Rehabilitation Specialized Group - GERF, São Paulo, SP 01239-040, Brazil. " Rehabilitation Sciences Program, Universidade Nove de Julho - UNINOVE, São Paulo, SP 02112-000, Brazil. ${ }^{5}$ NANTEN Healthy and Orthopaedic Institute, São Paulo, SP 01227-000, Brazil. ' La Trobe Sport and Exercise Medicine Research Centre, La Trobe University, Vic, Melbourne 3086, Australia. 'Human Movement of Science Department - Physical Therapy Course, Universidade Federal de São Paulo - UNIFESP, campus Baixada Santista, Santos, SP 11015-029, Brazil.

\section{Received: 7 May 2019 Accepted: 9 December 2019}

Published online: 27 December 2019

\section{References}

1. Pecina M, Bojanic I, Ivkovic A, Brcic L, Smoljanovic T, Seiwerth S. Patellar tendinopathy: histopathological examination and follow-up of surgical treatment. Acta Chir Orthop Traumatol Cechoslov. 2010;77(4):277-83.

2. Sharma P, Maffulli N. Biology of tendon injury: healing, modeling and remodeling. J Musculoskelet Neuronal Interact. 2006;6(2):181-90.

3. Reinking M. Tendinopathy in athletes. Phys Ther Sport. 2012;13(1):3-10.

4. Malliaras P, Cook J, Purdam C, Rio E. Patellar Tendinopathy: clinical diagnosis, load management, and advice for challenging case presentations. J Orthop Sports Phys Ther. 2015;45(11):887-98.

5. Lian OB, Engebretsen L, Bahr R. Prevalence of jumper's knee among elite athletes from different sports: a cross-sectional study. Am J Sports Med. 2005:33(4):561-7.

6. Zwerver J, Bredeweg SW, van den Akker-Scheek I. Prevalence of Jumper's knee among nonelite athletes from different sports: a cross-sectional survey. Am J Sports Med. 2011;39(9):1984-8.

7. Blazina ME, Kerlan RK, Jobe FW, Carter VS, Carlson GJ. Jumper's knee. Orthop Clin North Am. 1973:4(3):665-78.

8. Roels J, Martens M, Mulier JC, Burssens A. Patellar tendinitis (jumper's knee). Am J Sports Med. 1978;6(6):362-8.

9. Rasmussen OS. Sonography of tendons. Scand J Med Sci Sports. 2000;10(6): 360-4.

10. Cassel M, Baur H, Hirschmuller A, Carlsohn A, Frohlich K, Mayer F. Prevalence of Achilles and patellar tendinopathy and their association to intratendinous changes in adolescent athletes. Scand J Med Sci Sports. 2015;25(3):e310-8.

11. Malliaras $P$, Cook J. Changes in anteroposterior patellar tendon diameter support a continuum of pathological changes. Br J Sports Med. 2011;45(13): 1048-51.

12. Pearson SJ, Hussain SR. Region-specific tendon properties and patellar tendinopathy: a wider understanding. Sports Med. 2014;44(8):1101-12.

13. Witvrouw E, Lysens R, Bellemans J, Cambier D, Vanderstraeten G. Intrinsic risk factors for the development of anterior knee pain in an athletic population. A two-year prospective study. Am J Sports Med. 2000;28(4):480-9.

14. Malliaras $P$, Cook $J$, Kent $P$. Reduced ankle dorsiflexion range may increase the risk of patellar tendon injury among volleyball players. J Sci Med Sport. 2006;9(4):304-9.

15. Mendonca LD, Verhagen E, Bittencourt NF, Goncalves GG, Ocarino JM Fonseca ST. Factors associated with the presence of patellar tendon abnormalities in male athletes. J Sci Med Sport. 2016;19(5):389-94.

16. Scott A, Docking S, Vicenzino B, Alfredson H, Murphy RJ, Carr AJ, Zwerver J, Lundgreen K, Finlay O, Pollock N, et al. Sports and exercise-related tendinopathies: a review of selected topical issues by participants of the second international scientific Tendinopathy symposium (ISTS) Vancouver 2012. Br J Sports Med. 2013;47(9):536-44.

17. Torres R, Ferreira J, Silva D, Rodrigues E, Bessa IM, Ribeiro F. Impact of patellar Tendinopathy on knee proprioception: a cross-sectional study. Clin J Sport Med. 2017;27(1):31-6.

18. Cook U. Ten treatments to avoid in patients with lower limb tendon pain. Br J Sports Med. 2018:52(14):882.

19. Xu Y, Murrell GA. The basic science of tendinopathy. Clin Orthop Relat Res. 2008;466(7):1528-38.

20. Scattone Silva R, Ferreira AL, Nakagawa TH, Santos JE, Serrao FV. Rehabilitation of patellar Tendinopathy using hip extensor strengthening and landing-strategy modification: case report with 6-month follow-up. J Orthop Sports Phys Ther. 2015;45(11):899-909.

21. van der Windt DA, van der Heijden GJ, van den Berg SG, ter Riet G, de Winter AF, Bouter LM. Ultrasound therapy for musculoskeletal disorders: a systematic review. Pain. 1999;81(3):257-71.

22. Chipchase LS, Williams MT, Robertson VJ. A national study of the availability and use of electrophysical agents by Australian physiotherapists. Physiother Theory Pract. 2009;25(4):279-96.

23. Fu SC, Shum WT, Hung LK, Wong MW, Qin L, Chan KM. Low-intensity pulsed ultrasound on tendon healing: a study of the effect of treatment duration and treatment initiation. Am J Sports Med. 2008; 36(9):1742-9.

24. Fu SC, Hung LK, Shum WT, Lee YW, Chan LS, Ho G, Chan KM. In vivo lowintensity pulsed ultrasound (LIPUS) following tendon injury promotes repair during granulation but suppresses decorin and biglycan expression during remodeling. J Orthop Sports Phys Ther. 2010;40(7):422-9.

25. Cook JL, Rio E, Purdam CR, Docking SI. Revisiting the continuum model of tendon pathology: what is its merit in clinical practice and research? $\mathrm{Br} J$ Sports Med. 2016;50(19):1187-91.

26. Alexander LD, Gilman DR, Brown DR, Brown JL, Houghton PE. Exposure to low amounts of ultrasound energy does not improve soft tissue shoulder pathology: a systematic review. Phys Ther. 2010;90(1):14-25.

27. Warden SJ, Metcalf BR, Kiss ZS, Cook JL, Purdam CR, Bennell KL, Crossley KM. Low-intensity pulsed ultrasound for chronic patellar tendinopathy: a randomized, double-blind, placebo-controlled trial. Rheumatology (Oxford). 2008;47(4):467-71.

28. Cook JL, Purdam CR. Is tendon pathology a continuum? A pathology model to explain the clinical presentation of load-induced tendinopathy. $\mathrm{Br} J$ Sports Med. 2009;43(6):409-16

29. Mendonca LM, Leite HR, Zwerver J, Henschke N, Branco G, Oliveira VC. How strong is the evidence that conservative treatment reduces pain and improves function in individuals with patellar tendinopathy? A systematic review of randomised controlled trials including GRADE recommendations. Br J Sports Med. 2019. https://doi.org/10.1136/bjsports-2018-099747. [Epub ahead of print].

30. Kettunen JA, Kvist M, Alanen E, Kujala UM. Long-term prognosis for jumper's knee in male athletes. A prospective follow-up study. Am J Sports Med. 2002;30(5):689-92

31. Cook JL, Purdam CR. Rehabilitation of lower limb tendinopathies. Clin Sports Med. 2003;22(4):777-89.

32. Visentini PJ, Khan KM, Cook JL, Kiss ZS, Harcourt PR, Wark JD. The VISA score: an index of severity of symptoms in patients with jumper's knee (patellar tendinosis). Victorian Institute of Sport Tendon Study Group. J Sci Med Sport. 1998;1(1):22-8.

33. Edwards S, Steele JR, MCGhee DE, Beattie S, Purdam C, Cook JL. Landing strategies of athletes with an asymptomatic patellar tendon abnormality. Med Sci Sports Exerc. 2010;42(11):2072-80.

34. Wageck BB, de Noronha M, Lopes AD, da Cunha RA, Takahashi RH, Costa LO. Cross-cultural adaptation and measurement properties of the Brazilian Portuguese version of the Victorian Institute of Sport Assessment-Patella (VISA-P) scale. J Orthop Sports Phys Ther. 2013;43(3):163-71.

35. Mellor R, Bennell K, Grimaldi A, Nicolson P, Kasza J, Hodges P, Wajswelner H, Vicenzino B. Education plus exercise versus corticosteroid injection use versus a wait and see approach on global outcome and pain from gluteal tendinopathy: prospective, single blinded, randomised clinical trial. $\mathrm{Br} J$ Sports Med. 2018;52(22):1464-72.

36. Beyer R, Kongsgaard M, Hougs Kjaer B, Ohlenschlaeger T, Kjaer M, Magnusson SP. Heavy slow resistance versus eccentric training as treatment for Achilles Tendinopathy: a randomized controlled trial. Am J Sports Med. 2015:43(7):1704-11.

37. Bahr R, Fossan B, Loken S, Engebretsen L. Surgical treatment compared with eccentric training for patellar tendinopathy (Jumper's knee). A randomized, controlled trial. J Bone Joint Surg Am. 2006;88(8):1689-98.

38. Shomoto K, Takatori K, Morishita S, Nagino K, Yamamoto W, Shimohira T, Shimada T. Effects of ultrasound therapy on calcificated tendinitis of the shoulder. J Jpn Phys Ther Assoc. 2002;5(1):7-11.

39. Ebenbichler GR, Pieber K, Kainberger F, Funovics M, Resch KL. Long-term outcome of ultrasound therapy for calcific tendinitis of the shoulder: results of a RCT. PM R. 2016;8(9S):S156.

40. Mendonca LD, Ocarino JM, Bittencourt NFN, Macedo LG, Fonseca ST. Association of hip and Foot Factors with Patellar Tendinopathy (Jumper's knee) in athletes. J Orthop Sports Phys Ther. 2018;48(9):676-84. 
41. Zhang ZJ, Lee WC, Ng GYF, Fu SN. Isometric strength of the hip abductors and external rotators in athletes with and without patellar tendinopathy. Eur J Appl Physiol. 2018;118(8):1635-40.

42. Scattone Silva R, Nakagawa TH, Ferreira AL, Garcia LC, Santos JE, Serrao FV. Lower limb strength and flexibility in athletes with and without patellar tendinopathy. Phys Ther Sport. 2016;20:19-25.

43. van Ark M, Cook JL, Docking SI, Zwerver J, Gaida JE, van den Akker-Scheek I, Rio E. Do isometric and isotonic exercise programs reduce pain in athletes with patellar tendinopathy in-season? A randomised clinical trial. J Sci Med Sport. 2016;19(9):702-6.

44. Reiman MP, Bolgla LA, Loudon JK. A literature review of studies evaluating gluteus maximus and gluteus medius activation during rehabilitation exercises. Physiother Theory Pract. 2012;28(4):257-68.

45. Distefano LJ, Blackburn JT, Marshall SW, Padua DA. Gluteal muscle activation during common therapeutic exercises. J Orthop Sports Phys Ther. 2009; 39(7):532-40.

46. Fukuda TY, Rossetto FM, Magalhaes E, Bryk FF, Lucareli PR, de Almeida Aparecida Carvalho N. Short-term effects of hip abductors and lateral rotators strengthening in females with patellofemoral pain syndrome: a randomized controlled clinical trial. J Orthop Sports Phys Ther. 2010;40(11): 736-42.

47. Maciel Rabello L, Zwerver J, Stewart RE, van den Akker-Scheek I, Brink MS. Patellar tendon structure responds to load over a 7-week preseason in elite male volleyball players. Scand J Med Sci Sports. 2019;29(7):992-9.

48. Benitez-Martinez JC, Martinez-Ramirez P, Valera-Garrido F, Casana-Granell J, Medina-Mirapeix F. Comparison of pain measures between tendons of elite basketball players with different Sonographic patterns. J Sport Rehabil. 2019. p. 1-6.

49. Price DD, McGrath PA, Rafii A, Buckingham B. The validation of visual analogue scales as ratio scale measures for chronic and experimental pain Pain. 1983;17(1):45-56.

50. Purdam $C R$, Jonsson $P$, Alfredson $H$, Lorentzon $R$, Cook JL, Khan KM. A pilot study of the eccentric decline squat in the management of painful chronic patellar tendinopathy. Br J Sports Med. 2004;38(4):395-7.

51. Benedetti TRB, PdC A, Rodriguez-Añez CR, Mazo GZ, Petroski ÉL. Reprodutibilidade e validade do Questionário Internacional de Atividade Física (IPAQ) em homens idosos. Rev Bras Med Esporte. 2007;13:11-6.

52. Mann KJ, Edwards S, Drinkwater EJ, Bird SP. A lower limb assessment tool for athletes at risk of developing patellar tendinopathy. Med Sci Sports Exerc. 2013:45(3):527-33.

53. Richards DP, Ajemian SV, Wiley JP, Brunet JA, Zernicke RF. Relation between ankle joint dynamics and patellar tendinopathy in elite volleyball players. Clin J Sport Med. 2002;12(5):266-72.

54. Bisseling RW, Hof AL, Bredeweg SW, Zwerver J, Mulder T. Relationship between landing strategy and patellar tendinopathy in volleyball. $\mathrm{Br} J$ Sports Med. 2007;41(7):e8.

55. Dingenen B, Malfait B, Vanrenterghem J, Verschueren SM, Staes FF. The reliability and validity of the measurement of lateral trunk motion in twodimensional video analysis during unipodal functional screening tests in elite female athletes. Phys Ther Sport. 2014;15(2):117-23.

56. Bell-Jenje T, Olivier B, Wood W, Rogers S, Green A, McKinon W. The association between loss of ankle dorsiflexion range of movement, and hip adduction and internal rotation during a step down test. Man Ther. 2016;21:256-61.

57. Jones D, Tillman SM, Tofte K, Mizner RL, Greenberg S, Moser MW, Chmielewski TL. Observational ratings of frontal plane knee position are related to the frontal plane projection angle but not the knee abduction angle during a step-down task. J Orthop Sports Phys Ther. 2014:44(12):973-8.

58. Nilstad A, Andersen TE, Kristianslund E, Bahr R, Myklebust G, Steffen K, Krosshaug T. Physiotherapists can identify female football players with high knee valgus angles during vertical drop jumps using real-time observational screening. J Orthop Sports Phys Ther. 2014;44(5):358-65.

59. Fernandes de Jesus J, de Almeida NA, Bezerra Nakaoka G, Curcio Dos Reis A, Fukuda TY, Fernandes Bryk F. Kinesio taping effect on quadriceps strength and lower limb function of healthy individuals: A blinded, controlled, randomized, clinical trial. Phys Ther Sport. 2016;18:27-31.

60. Jesus JF, Bryk FF, Moreira VC, Nakaoka GB, Reis AC, Lucareli PRG. Gluteus Maximus inhibition in proximal hamstring tendinopathy. MedicalExpress (São Paulo, online). 2015;2(4):150406.

61. Magalhaes E, Fukuda TY, Sacramento SN, Forgas A, Cohen M, Abdalla RJ. A comparison of hip strength between sedentary females with and without patellofemoral pain syndrome. J Orthop Sports Phys Ther. 2010; 40(10):641-7.

62. Stark T, Walker B, Phillips JK, Fejer R, Beck R. Hand-held dynamometry correlation with the gold standard isokinetic dynamometry: a systematic review. PM R. 2011;3(5):472-9.

63. van Wilgen $P$, van der Noord $R, Z$ Zwerver J. Feasibility and reliability of pain pressure threshold measurements in patellar tendinopathy. J Sci Med Sport. 2011;14(6):477-81.

64. Ring EF, Ammer K. Infrared thermal imaging in medicine. Physiol Meas. 2012;33(3):R33-46.

65. Mangine RE, Siqueland KA, Noyes FR. The use of thermography for the diagnosis and management of patellar tendinitis. J Orthop Sports Phys Ther 1987;9(4):132-40.

66. Zaproudina N, Varmavuo V, Airaksinen O, Narhi M. Reproducibility of infrared thermography measurements in healthy individuals. Physiol Meas. 2008;29(4):515-24

67. Hildebrandt C, Raschner C, Ammer K. An overview of recent application of medical infrared thermography in sports medicine in Austria. Sensors (Basel). 2010;10(5):4700-15.

68. Sanz-Lopez F, Martinez-Amat A, Hita-Contreras F, Valero-Campo C, Berzosa C. Thermographic assessment of eccentric overload training within three days of a running session. J Strength Cond Res. 2016:30(2):504-11.

69. McLoughlin RF, Raber EL, Vellet AD, Wiley JP, Bray RC. Patellar tendinitis: MR imaging features, with suggested pathogenesis and proposed classification. Radiology. 1995;197(3):843-8.

70. Tsehaie J, Poot DHJ, Oei EHG, Verhaar JAN, de Vos RJ. Value of quantitative MRI parameters in predicting and evaluating clinical outcome in conservatively treated patients with chronic midportion Achilles tendinopathy: a prospective study. J Sci Med Sport. 2017;20(7):633-7.

71. Williams H, Jones SA, Lyons C, Wilson C, Ghandour A. Refractory patella tendinopathy with failed conservative treatment-shock wave or arthroscopy? J Orthop Surg. 2017;25(1):2309499016684700.

72. Schulz KF, Altman DG, Moher D, Group C. CONSORT 2010 statement: updated guidelines for reporting parallel group randomised trials. BMJ. 2010;340:c332.

73. Elkins MR, Moseley AM. Intention-to-treat analysis. J Physiother. 2015;61(3): $165-7$

74. Gaida JE, Cook JL, Bass SL, Austen S, Kiss ZS. Are unilateral and bilateral patellar tendinopathy distinguished by differences in anthropometry, body composition, or muscle strength in elite female basketball players? $\mathrm{Br} J$ Sports Med. 2004;38(5):581-5.

75. McAuliffe S, McCreesh K, Culloty F, Purtill H, O'Sullivan K. Can ultrasound imaging predict the development of Achilles and patellar tendinopathy? A systematic review and meta-analysis. Br J Sports Med. 2016:50(24):1516-23.

76. Mersmann F, Pentidis N, Tsai MS, Schroll A, Arampatzis A. Patellar tendon strain associates to tendon structural abnormalities in adolescent athletes. Front Physiol. 2019;10:963.

77. Rio E, Kidgell D, Moseley GL, Gaida J, Docking S, Purdam C, CookJ. Tendon neuroplastic training: changing the way we think about tendon rehabilitation: a narrative review. Br J Sports Med. 2016;50(4):209-15.

78. Chan AK, Myrer JW, Measom GJ, Draper DO. Temperature changes in human patellar tendon in response to therapeutic ultrasound. J Athl Train. 1998:33(2):130-5.

79. Downing DS, Weinstein A. Ultrasound therapy of subacromial bursitis. A double blind trial. Phys Ther. 1986:66(2):194-9.

80. Lehmann JF, Masock AJ, Warren CG, Koblanski JN. Effect of therapeutic temperatures on tendon extensibility. Arch Phys Med Rehabil. 1970:51(8):481-7.

81. Abramson DI, Burnett C, Bell Y, Tuck S Jr, Rejal H, Fleischer CJ. Changes in blood flow, oxygen uptake and tissue temperatures produced by therapeutic physical agents. Am J Phys Med. 1960;39:51-62.

82. Robertson VJ, Baker KG. A review of therapeutic ultrasound: effectiveness studies. Phys Ther. 2001;81(7):1339-50.

83. Best TM, Moore B, Jarit P, Moorman CT, Lewis GK. Sustained acoustic medicine: wearable, long duration ultrasonic therapy for the treatment of tendinopathy. Phys Sportsmed. 2015;43(4):366-74

\section{Publisher's Note}

Springer Nature remains neutral with regard to jurisdictional claims in published maps and institutional affiliations. 